\title{
Participants and Non-participants in the Scand-Ankle study - An alcohol cessation intervention at the time of fracture surgery
}

\author{
Marianne Aalykke ${ }^{1}$, Erika Wernheden ${ }^{1}$, Bolette Pedersen ${ }^{1,2}$, Julie WM Egholm ${ }^{1,3}$, Bjørn L \\ Madsen ${ }^{4}$, Jes B Lauritzen ${ }^{5}$, Hanne Tønnesen ${ }^{1,2}$
}

\begin{abstract}
Background Clinical experience indicates that patients declining participation in randomised clinical trials (RCTs) at the time of surgery are older, less healthy and of lower social status than patients accepting to participate, compromising the external validity of the RCT and bringing the non-participants in higher risk at surgery. To our knowledge, no studies exist on patients with hazardous alcohol consumption who decline participation in RCTs at the time of surgery. The aim was to compare characteristics of the participants and non-participants in the Scand-Ankle RCT.

Method The Scand-Ankle study is a RCT that investigates the effect of a 6-week gold standard alcohol intervention (GSP-A) on postoperative complications in patients drinking $>21$ units/week and undergoing ankle fracture surgery. This study included eligible patients that declined to participate in the Scand-Ankle RCT but gave informed consent to follow-up in their medical record ( $\mathrm{N}=67)$. Their perioperative patient characteristics were obtained from their medical record and the characteristics were compared to the patients enrolled in the study so far ( $\mathrm{N}=61)$.

Results The baseline characteristics showed no differences between participants and non-participants, but some tendencies towards the non-participants being older, having severak co-morbidities and higher ASA scores. None of the variables of interest in relation to postoperative complications (age over 70 years, comorbidity, daily smoking, ASA scores, fracture type, BMI, alcohol) were significant predictors for non-participation.
\end{abstract}

Conclusion No differences were found between participants and non-participants and no patient characteristics could significantly predict participation.

doi.org/10.29102/clinhp.15008

\section{About the AUTHORS}

${ }^{1}$ WHO-CC, Bispebjerg/ Frederiksberg University Hospital, Denmark

${ }^{2}$ Clinical Health Promotion Centre, Lund University, Sweden

${ }^{3}$ University of Southern Denmark, Hospital of Southern Jutland, Denmark

${ }^{4}$ Orthopedic Department, Hvidovre Hospital, Denmark 5Orthopedic Department, Bispebjerg Hospital, Denmark

Contact:

Marianne Aalykke marianne.aalykke@gmail.com

Clin Health Promot 2015; 5:49-53

\section{Introduction}

Patients declining participation in randomized clinical trials (RCT) is a wellknown concern, and a low recruitment rate can cause selection bias and compromise the external validity $(1,2)$. The patients who participate in clinical trials are typically considered to be healthier, younger and of higher social status (3). A review (by Abraham et al.) found that four different surgical RCTs had ambiguous conclusions when comparing participants with non-participants, though a tendency was found towards the nonparticipants being older, less well-educated, likely to be more ill, and have their condition interfere more with their daily life. This review also showed that the three most common reasons for patients not to participate in surgical RCTs were: preference for one form of treatment, dislike of the idea of randomization, and the potential for increased demands of the patients (4).

We know of no existing studies on the comparison between participants and non-participants in an alcohol cessation intervention at the time of surgery.

The purpose of this study was to describe baseline characteristics of the non-participants and the participants, as well as to investigate predictors of being non-participant compared to participant in the multicenter Scand-Ankle RCT regarding alcohol cessation intervention in acute ankle fracture surgery. 


\section{Research and Best Practice}

Based on our own clinical experience and earlier studies, our hypothesis was that patients with hazardous alcohol consumption that declined participation in the ScandAnkle trial had more preoperative risk factors than participants, making them subject to an even higher risk of developing postoperative complications.

\section{Method}

\section{Study design}

This is a descriptive study based on the non-participants and participants of the Scand-Ankle RCT.

\section{The Scand-Ankle RCT}

The Scand-Ankle trial is an ongoing RCT investigating the effect of a new 6-week gold standard alcohol intervention (GSP-A) on postoperative complications, alcohol intake and cost-effectiveness in hazardous drinking patients undergoing ankle fracture surgery. The study is conducted at two university hospitals in Copenhagen.

Patients are eligible for the study if the timeline followback (5) identifies an alcohol consumption in the patient of $\geq 21$ units/week (one unit equals $12 \mathrm{~g}$ of ethanol) on average during the past three months, undergo ankle fracture surgery with internal fixation, and give informed consent within 36 hours from admission. Patients are excluded if they are under 18 years old or are unable to give informed consent, are pregnant or breastfeeding, are allergic to Disulfiram or Benzodiazepines, have previously experienced delirium or cramps during abstinence, have multiple or pathological fractures, or have an American Society of Anaesthesiologists physical status classification (ASA scores) $\geq 4$ (6) or fulminant heart or liver insufficiency.

The intervention group receives the 6-week GSP-A consisting of a structured patient education program with weekly meetings ( 5 in total), as well as Disulfiram (200 mg x 2 per week) and B-vitamin and Thiamin.

The control group receives the orthopedic department's standard care for patients with ankle fracture and hazardous alcohol intake.

All participants are free to seek alcohol abuse treatment outside of the GSP-A. Both groups are followed up at 6 weeks where the patients would attend their routine Xray and clinical control at the department.

\section{Inclusion and exclusion criteria}

Patients were included in this study if they were enrolled in the Scand-Ankle trial or were eligible but declined to participate in the Scand-Ankle trial at Hvidovre and Bispebjerg hospitals from April 2010 to December 2013.
Patients were excluded if they declined to participate in the Scand-Ankle trial or did not give informed consent to follow-up via their medical record material.

\section{Data registration/Outcomes}

The primary outcomes were a comparison of baseline characteristics between participants and non-participants and predictors of being non-participant compared to participant.

The baseline characteristics were: age, sex, body mass index (BMI), daily smoking, alcohol (units per week), employment, living alone, ASA score (ASA score 1: a normal healthy patient; ASA score 2: a patient with a mild systemic disease; ASA score 3: a patient with a severe systemic disease), comorbidity (diagnosed: lung disease, cardiovascular disease, liver disease, psychiatric disorders, neurologic disorders, musculoskeletal diseases, diabetes) and fracture type. The predictors were all patient characteristic variables at risk of having a relation to the complication rate: age $>70$ years(7-10), daily smoking(9), ASA scores (6), comorbidity (lung, liver, heart diseases and diabetes)(6), alcohol $\geq 35$ units/week (11), fracture type (12) and BMI (13). Only patient risk factors at baseline were chosen as predictors because the patients often were asked to participate in the trial preoperatively. The data of the non-participants and participants were collected from the patients' medical record material.

\section{Analysis}

The patient characteristics were described using median and range for continuous data and number and percentages for categorical data. To detect if any complication rate risk factor could predict participation, univariate analyses (Chi-Square and Fisher's exact test) and multivariate analyses (logistic regression) were performed on the predictive variables. Analyses were performed in in IBM SPSS v. 19 and Excel 2010.

\section{Ethics}

The Scand-Ankle protocol was registered in ClinicalTrials.gov (id: NCTo0986791). The project has been approved by the Danish Scientific Ethical Committee System (CVK: 0908664) and the Danish Data Protection Agency (28th of July 2009). The non-participant group in this study gave informed consent to follow-up via their medical record material.

\section{Results}

Study groups

A total of 141 patients were eligible for the Scand-Ankle trial in the period April 2010 to December 2013 at 


\section{Research and Best Practice}

Hvidovre and Bispebjerg hospitals. 77 patients declined to participate and 64 were included in the Scand-Ankle trial. One withdrew consent after inclusion and two had their operations cancelled. Out of the 77 patients declining participation, 67 gave informed consent to follow-up via their medical record material. A total of 67 non-participants and 61 participants were included in this study (Figure I).

Figure 1 Trial profile

Eligble patients at Bisbebjerg

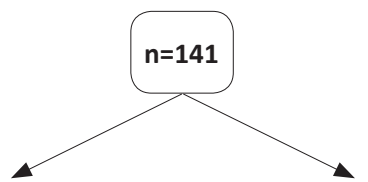

Included in the

Declined the

Scand-Ankle trial

Scand-Ankle trial
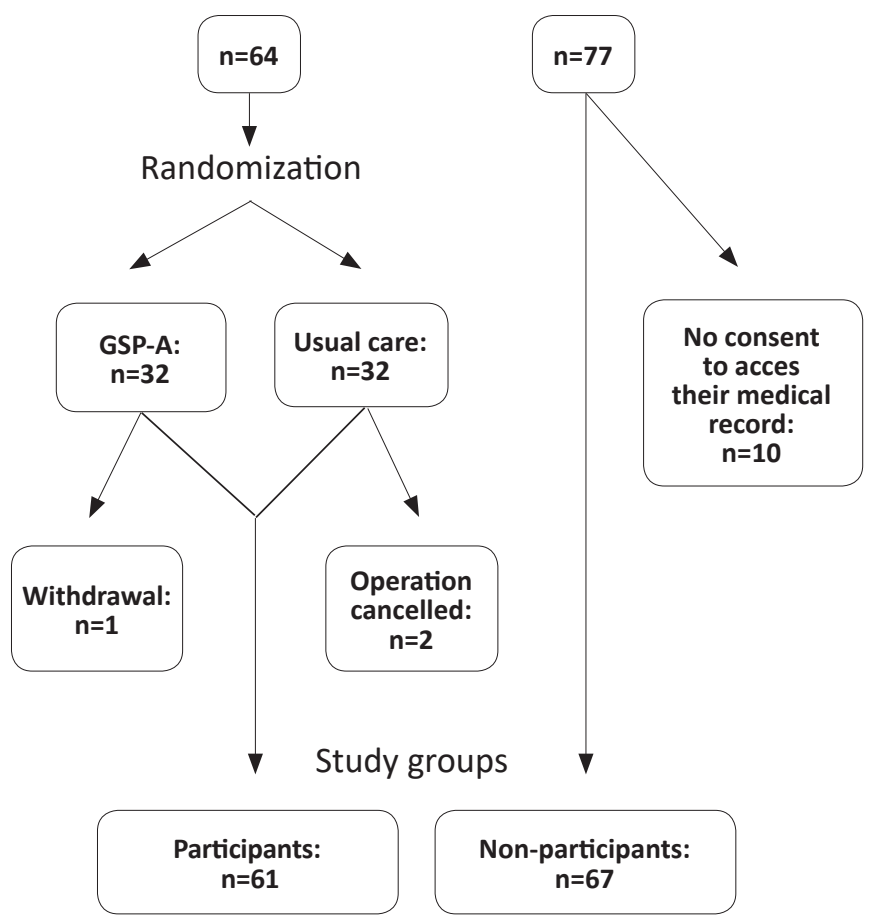

Baseline characteristics of participants vs. nonparticipants

Table 1 shows the baseline characteristics of the two groups. There were no differences between participants and non-participants, but some tendencies towards the non-participants being older, having more co-morbidity and higher ASA scores.

In the non-participant group 55\% had co-morbidity, 57\% had ASA score 2 and $9 \%$ had ASA score 3. In the participant group 44\% had comorbidity, 49\% had ASA score 2 and $5 \%$ had ASA score 3 .

\begin{tabular}{|c|c|c|c|}
\hline \multirow[b]{2}{*}{ Age } & $\begin{array}{c}\text { Non-participants } \\
\quad(\mathrm{N}=67)\end{array}$ & \multicolumn{2}{|c|}{$\begin{array}{l}\text { Participants } \\
\qquad(\mathrm{N}=61)\end{array}$} \\
\hline & $57(19-82)$ & 50 & $(20-78)$ \\
\hline Sex (men) & $50 \quad(75 \%)$ & 40 & $(66 \%)$ \\
\hline BMI (kg/m2) & $26(17-46)$ & 26 & $(17-37)$ \\
\hline Daily smoking & $43 \quad(64 \%)$ & 39 & $(64 \%)$ \\
\hline Employed* & $23 \quad(41 \%)$ & 25 & $(41 \%)$ \\
\hline Living alone & $39 \quad(58 \%)$ & 35 & $(58 \%)$ \\
\hline Alcohol (units per week) ${ }^{* *}$ & $28(14-210)$ & 30 & $(0-126)$ \\
\hline Patients with co-morbidity ${ }^{* * *}$ & $37 \quad(55 \%)$ & 27 & $(44 \%)$ \\
\hline Lung disease & $4 \quad(6 \%)$ & 6 & $(10 \%)$ \\
\hline Cardiovascular disease & $17 \quad(25 \%)$ & 11 & $(18 \%)$ \\
\hline Diabetes & $(3 \%)$ & 1 & $(2 \%)$ \\
\hline Liver disease & $(4 \%)$ & 2 & (3\%) \\
\hline Minor psychiatric disorder & $(9 \%)$ & 15 & $(25 \%)$ \\
\hline Neurologic disorder & (9\%) & 3 & (5\%) \\
\hline Musculoskeletal disease & $7 \quad(10 \%)$ & 4 & $(7 \%)$ \\
\hline Other diseases & $(7 \%)$ & 8 & $(13 \%)$ \\
\hline \multicolumn{4}{|l|}{ ASA score } \\
\hline 1 & $23 \quad(34 \%)$ & 28 & $(46 \%)$ \\
\hline 2 & $38 \quad(57 \%)$ & 30 & (49\%) \\
\hline 3 & $(9 \%)$ & 8 & $(13 \%)$ \\
\hline \multicolumn{4}{|l|}{ Fracture type } \\
\hline Unimalleoalar & (51\%) & 26 & $(43 \%)$ \\
\hline Bimalleolar & $24 \quad(36 \%)$ & 27 & $(44 \%)$ \\
\hline Trimalleolar & $9 \quad(13 \%)$ & 8 & $(13 \%)$ \\
\hline
\end{tabular}

Data are numbers (\%) or medians (range). "11 non-participants had unknown employment. "*All patients had an average alcohol intake $\geq 21$ units/week in the last three months. ${ }^{* *}$ Diagnosed musculoskeletal - , lung -, liver -, heart disease, diabetes, psychiatric -, neurologic disorder and other diseases.

Predictors of non-participation compared to participation

Table II shows the variables of interest: age over 70 years, comorbidity (heart, lung, liver disease and diabetes), daily smoking, ASA scores, fracture type, BMI, and alcohol. None of the variables were significant predictors for non-participation in neither the uni- or multivariate analyses.

There was a tendency towards ASA score 3 being a predictor of non-participation $(\mathrm{OR}=3,0 ; \mathrm{CI}=0,51-18,12)$.

\section{Discussion}

Baseline characteristics, participants vs. non participants

Contrary to our hypothesis, the non-participants did not have more preoperative risk factors than the participants. 


\section{Research and Best Practice}

Table 2 Predictors of non-participation compared to participation

\begin{tabular}{|c|c|c|c|c|c|}
\hline \multirow[b]{3}{*}{ Age ( $\leq 70$ vs. $>70$ ) } & \multicolumn{3}{|c|}{ Univariate analysis } & \multicolumn{2}{|c|}{ Multivariate analysis } \\
\hline & \multicolumn{2}{|c|}{ Odds ratio $(95 \% \mathrm{Cl})$} & \multirow{2}{*}{$\frac{\text { p-value }}{0.766}$} & Odds ratio (95\%) & \multirow{2}{*}{$\begin{array}{c}p \text {-value } \\
0.846\end{array}$} \\
\hline & 1.31 & $(0.39-4.36)$ & & $1.14 \quad(0.3-4.32)$ & \\
\hline Comorbidity* ( - vs. + ) & 1.4 & $(0.63-3.05)$ & 0.396 & $1.21 \quad(0.44-3.36)$ & 0.712 \\
\hline Daily smoking (- vs. +) & 1.01 & $(0.49-2.08)$ & 0.977 & $1.19 \quad(0.53-2.68)$ & 0.675 \\
\hline \multicolumn{6}{|l|}{ ASA score } \\
\hline (1 vs. 2 ) & 1.54 & $(0.74-3.20)$ & 0.244 & $1.37 \quad(0.55-3.42)$ & 0.495 \\
\hline (1 vs. 3 ) & 2.44 & $(0.55-10.82)$ & 0.292 & $3.0(0.51-18.12)$ & 0.224 \\
\hline \multicolumn{6}{|l|}{ Fracture type } \\
\hline Unimalleolar vs. bimalleolar & 0.68 & $(0.32-1.44)$ & 0.313 & $0.55 \quad(0.24-1.26)$ & 0.156 \\
\hline Unimalleolar vs. trimalleolar & 0.86 & $(0.29-2.54)$ & 0.785 & $(0.2-2.12)$ & 0.495 \\
\hline \multicolumn{6}{|l|}{ BMI } \\
\hline Normal vs. underweight & 1.82 & $(0.15-21.61)$ & 1 & $1.14(0.08-16.24)$ & 0.923 \\
\hline Normal vs. overweight & 0.78 & $(0.35-1.76)$ & 0.548 & $0.61 \quad(0.25-1.49)$ & 0.281 \\
\hline Normal vs. obese & 1.21 & $(0.46-3.17)$ & 0.695 & $1.09 \quad(0.39-3.07)$ & 0.866 \\
\hline Alcohol (21-35 vs. $\geq 35$ units/week) & 0.69 & $(0.33-1.42)$ & 0.309 & $0.62 \quad(0.27-1.42)$ & 0.256 \\
\hline
\end{tabular}

*Diagnosed lung, liver, heart, disease or diabetes.

There is an inconsistency between the number of patients with ASA score 2, ASA score 3 and the number of patients with co-morbidity in both groups. A possible reason could be that the ASA score was evaluated by an anaesthesiologist and sometimes a high alcohol consumption alone gave the patient an ASA score of 2 instead of 1 . In the classification system, an exact alcohol amount is not defined, so it remains up to each anaesthesiologist to determine how large a weekly alcohol intake it takes to classify the patient as ASA score 2.

To our knowledge, this is the first study to compare participants and non-participants in an alcohol cessation intervention RCT at the time of surgery. Sparse literature is available on comparing participants with nonparticipants in lifestyle intervention RCTs at the time of surgery, but some studies have shown that non-participants in psychosocial intervention RCTs following surgery had lower socioeconomic status than the participants $(14 ; 15)$. One of these studies also found that the non-participants were older (15) and the other found no difference in age (14). In our study, there is a tendency towards the non-participants being older.

The non-participants' alcohol intake is not different from that of the participants, indicating that patients with hazardous alcohol consumption might have the same reasons for declining participation in a RCT as patients with an alcohol use not exceeding the recommended limits.

\section{Predictors of non-participation compared to participation}

No patient characteristics that were related to the complication rate could predict participation. This indicates that at the time of inclusion, the non-participants did not have a higher risk at surgery compared to the participants. The Scand-Ankle participants appear to be representative of the patient population which we wished to investigate, in relation to factors relevant to the outcome i.e. postoperative complications.

\section{Bias and limitations}

The strength of this study is that almost all of the nonparticipants gave informed consent to follow-up via their medical record.

A limitation to this study is the missing information about the patient's income, residence and educational level. Differences in these parameters could affect the applicability of the Scand-Ankle trial. Living alone and employment are socioeconomic factors present in this study and no differences in these parameters were found. A bias in this study is the missing registration of open fractures, which is related to a higher complication rate $(7,8)$. Another bias is the risk of a type 2 error because of the number of participants. There was a tendency towards ASA score 3 (vs. ASA score 1) being a predictor of non-participation, but it would demand a study group of 328 patients to show a significant association. 


\section{Research and Best Practice}

\section{Perspectives}

A survey about the patient's reasons for not participating could be of great interest especially for alcohol intervention studies in relation to surgery. An interview study about patient opinion was performed before the start of the Scand-Ankle trial and showed that nearly half of the patients were ready to participate in an alcohol intervention and all the patients found the Scand-Ankle trial to be relevant in relation to surgery. Interviews of reasons were not performed in the study (16). The challenge of patient inclusion in lifestyle interventions in an acute surgical setting is shown through the interview study, the inclusion rate in the Scand-Ankle trial and experiences from a smoking cessation intervention at the time of ankle fracture surgery with an inclusion rate of $35 \%$ (17).

Once the Scand-Ankle study is finalised, the complication rate among the Scand-Ankle patients will be evaluated, and it would be relevant to compare the complication rate of the non-participant with the Scand-Ankle control group and intervention group, since it has been suggested that there exists a beneficial "trial effect" by participating in RCTs $(18 ; 19)$.

The GSP-A has already shown to be cost-effective (20) and significantly more patients in the intervention group (58\%) were abstainers than in the control group (13\%) (20). Another result from the Scand-Ankle study is that the GSP-A had no effect on smoking cessation or any other lifestyle risk factor, suggesting that a potential effect on postoperative complications is related to the alcohol intake.

\section{Conclusion}

No differences were found between participants and non-participants and no patient characteristics related to the complication rate could predict participation.

\section{Acknowledgement}

Ina Stark, Anne-Birgitte Larsen \& Troels Riis for clinical assistance, and Mette Rasmussen for technical assistance.

\section{Contribution details}

\section{Conception and design: MA \& HT}

Acquisition, analysis and/or interpretation of data: All

Drafting the article: MA

Revising and approving the article: All

\section{Competing interests \\ None declared}

\section{References}

(1) Britton A, McKee M, Black N, McPherson K, Sanderson C, Bain C. Threats to applicability of randomised trials: exclusions and selective participation. J Health Serv Res Policy 1999; 4:112-21.

(2) Charlson ME, Horwitz RI. Applying results of randomised trials to clinical practice: impact of losses before randomisation. Br Med J (Clin Res Ed) 1984; 289:1281-4.

(3) Mant D. Can randomised trials inform clinical decisions about individual patients? Lancet 1999; 9154:353.

(4) Abraham NS, Young JM, Solomon MJ. A systematic review of reasons for nonentry of eligible patients into surgical randomized controlled trials. Surgery. 2006; 139:469-83.

(5) Sobell MB, Sobell LC, Klajner F, Pavan D, Basian E. The reliability of a timeline method for assessing normal drinker college students' recent drinking history: Utility for alcohol research. Addict Behav 1986; 11:149-61.

(6) ASA Physical Status Classification System. American Society of Anesthesiologists. http://www.asahq.org/ /media/sites/asahq/files/public/resources/standards-guidelines/asa-physical-status-classification-system.pdf.

(7) SooHoo NF, Eagan M, Krenek L, Zingmond DS. Incidence and factors predicting pulmonary embolism and deep venous thrombosis following surgical treatment of ankle fractures. Foot Ankle Surg 2011; 17:259-62.

(8) SooHoo NF, Krenek L, Eagan MJ, Gurbani B, Ko CY, Zingmond DS. Complication rates following open reduction and internal fixation of ankle fractures. J Bone Joint Surg Am 2009; 91:1042-9.

(9) Ovaska MT, Mäkinen TJ, Madanat R, Huotari K, Vahlberg T, Hirvensalo E, et al. Risk factors for deep surgical site infection following operative treatment of ankle fractures. J Bone Joint Surg Am 2013; 95:348-53.

(10) Srinivasan CM., Moran CG. Internal fixation of ankle fractures in the very elderly. Injury 2001; 32:559-63.

(11) Tønnesen H, Pedersen A, Jensen MR, Møller A, Madsen JC. Ankle fractures and alcoholism. The influence of alcoholism on morbidity after malleolar fractures. J Bone Joint Surg Br 1991; 73:511-3.

(12) Dodson NB, Ross AJ, Mendicino RW, Catanzariti AR. Factors affecting healing of ankle fractures. J Foot Ankle Surg 2013; 52:2-5.

(13) Sadr Azodi O, Bellocco R, Eriksson K, Adami J. The impact of tobacco use and body mass index on the length of stay in hospital and the risk of post-operative complications among patients undergoing total hip replacement. J Bone Joint Surg Br 2006; 88:1316-20.

(14) Boesen E, Boesen S, Christensen S, Johansen C. Comparison of participants and non-participants in a randomized psychosocial intervention study among patients with malignant melanoma. Psychosomatics 2007; 48:510-6.

(15) Sears SR, Stanton AL, Kwan L, Krupnick JL, Rowland JH, Meyerowitz BE, et al. Recruitment and retention challenges in breast cancer survivorship research: results from a multisite, randomized intervention trial in women with early stage breast cancer. Cancer Epidemiol Biomarkers Prev 2003; 12:1087-90.

(16) Pedersen B, Alva-Jørgensen P, Raffing R, Tønnesen H. Fractures and alcohol abuse - patient opinion of alcohol intervention. Open Orthop J 2011; 5:7-12. (17) Nåsell H, Adami J, Samnegård E, Tønnesen H, Ponzer S. Effect of smoking cessation intervention on results of acute fracture surgery: a randomized controlled trial. J Bone Joint Surg. Am 2010; 92:1335-42

(18) Braunholtz DA, Edwards SJ, Lilford RJ. Are randomized clinical trials good for us (in the short term)? Evidence for a "trial effect". J Clin Epidemiol 2001; 54:21724.

(19) West J, Wright J, Tuffnell D, Jankowicz D, West R. Do clinical trials improve quality of care? A comparison of clinical processes and outcomes in patients in a clinical trial and similar patients outside a trial where both groups are managed according to a strict protocol. Qual Saf Health Care 2005; 14:175-8.

(20) Pedersen B. Scand-Ankle: Cost-effectiveness of Alcohol Cessation Intervention in Acute Fracture Surgery - PHD Thesis. Clin Heal Promot - Res Best Pract patients, Staff community 2014; 4. 